\title{
A prospective multicentre study to evaluate the consistency of the IHS diagnostic criteria, the usefulness of brain MRI for the diagnosis, follow-up and treatment management, and the outcome after high dosage 6-methylprednisolone therapy, in subjects with Tolosa-Hunt syndrome
}

\author{
Silvia Colnaghi - Maurizio Versino - Enrico Marchioni - Cristina Tassorelli • \\ Stefano Bastianello - Giorgio Sandrini - Giuseppe Nappi
}

Received: 24 February 2010/Accepted: 2 March 2010/Published online: 25 March 2010

(C) Springer-Verlag 2010

\section{Dear Editor,}

As discussed in a previous review [1], several reasons suggest the need for a revision of diagnostic criteria of the International Headache Classification (IHS) [2] for Tolosa-Hunt syndrome (THS). First of all evidence is lacking on the most appropriate steroid treatment, secondly several reports suggest that neurological signs persist beyond the time limit defined by the IHS criteria. Furthermore, specific MRI techniques are necessary for detecting the inflammatory tissue, which can extend beyond the cavernous sinus and the orbit.

Since data available from the literature mostly derive from case reports which differ in terms of treatment

Electronic supplementary material The online version of this article (doi:10.1007/s10194-010-0206-3) contains supplementary material, which is available to authorized users.

S. Colnaghi $(\bowtie) \cdot M$. Versino $\cdot$ E. Marchioni - C. Tassorelli ·

G. Sandrini - G. Nappi

Headache Science Centre, IRCCS National Neurological Institute C. Mondino, Pavia, Italy

e-mail: silvia.colnaghi@mondino.it

S. Colnaghi - M. Versino - E. Marchioni - C. Tassorelli

G. Sandrini - G. Nappi

UCADH University Centre for Adaptive Disorders

and Headache, University of Pavia, Pavia, Italy

S. Colnaghi - M. Versino - C. Tassorelli - S. Bastianello ·

G. Sandrini

Department of Neurological Sciences,

University of Pavia, Pavia, Italy

S. Bastianello

Division of Neuroradiology, IRCCS National Neurological

Institute C. Mondino, Pavia, Italy

G. Nappi

Department of Neurology and ENT,

University 'La Sapienza', Roma, Italy schedules, MRI techniques, and follow-up strategies, IHS diagnostic criteria cannot be improved by adopting an evidence-based methodology. Thus, we are promoting a non-profit multicentre study aimed at revising THS diagnostic criteria, considering (1) the clinical and MRI characteristics of the disease at presentation, after a standardized steroid treatment, and during an 8-month followup from the time symptoms, signs and MRI normalize, (2) the occurrence of relapses after treatment discontinuation, and (3) the effect of the lesion site on the outcome.

Any participating centre will be asked to follow a simple management protocol based on serial MRI evaluations. All data will be recorded in case report forms provided by the Coordinator Centre, and no migration of investigators or patients is needed.

The study has received approval from the Ethical Committee of the Coordinator Centre IRCCS "National Neurological Institute C. Mondino" Foundation. All contributors will appear as co-authors of every presentation and/or scientific publication of partial or complete results of the study.

The readers of The Journal of Headache and Pain who are willing to participate can download the Study Summary, a Schematic Diagram and a Time and Event Schedule (available online as supplementary material), and can ask the corresponding author for complete documentation of the study.

Conflict of interest None.

\section{References}

1. Colnaghi S, Versino M, Marchioni E, Pichiecchio A, Bastianello S, Cosi V, Nappi G (2008) ICHD-II diagnostic criteria for TolosaHunt syndrome in idiopathic inflammatory syndromes of the orbit and/or the cavernous sinus. Cephalalgia 28(6):577-584

2. The International Classification of Headache Disorders ICHD-II (2004) Cephalalgia 24 (Suppl. 1):131 